\title{
Radial and Nonradial Solutions for a Semilinear Elliptic System of Schrödinger Type
}

\author{
By \\ Dragoş-Pătru CoveI ${ }^{1}$ \\ (Constantin Brâncuşi University of Târgu-Jiu and West University of Timişoara, România)
}

\begin{abstract}
In this article we consider the system of equations $\Delta u_{i}=p_{i}(x) f_{i}\left(u_{1}, \ldots, u_{d}\right)$ for $i=1, \ldots, d$ on $\boldsymbol{R}^{N}, N \geq 3$ and $d \in\{1,2,3,4, \ldots\}$. We prove that the considered system has a bounded positive entire solution under some conditions on $p_{i}$ and $f_{i}$. Also, we give a necessary condition as well as a sufficient condition for a positive radial solution to be large. The method of proving theorems is essentially based on a successive approximation. Furthermore, a non-radially symmetric solution is obtained by using a lower and upper solution method.

Key Words and Phrases. Entire solution, Large solution, Elliptic system.

2010 Mathematics Subject Classification Numbers. 35J61, 35J91.
\end{abstract}

\section{Introduction}

In this paper we study the existence of solutions for the semilinear elliptic system

$$
\left\{\begin{array}{l}
\Delta u_{1}=p_{1}(x) f_{1}\left(u_{1}, \ldots, u_{d}\right), \\
\cdots \\
\Delta u_{d}=p_{d}(x) f_{d}\left(u_{1}, \ldots, u_{d}\right),
\end{array} \quad \text { in } \boldsymbol{R}^{N},\right.
$$

where $N \geq 3, d \geq 1$, and the functions $p_{j}, f_{j}(j=1, \ldots, d)$ are supposed to satisfy the following hypothesis:

(P1) $p_{j}: \boldsymbol{R}^{N} \rightarrow[0, \infty)$ are locally Hölder continuous functions of exponent $\alpha \in(0,1)$;

(C1) $f_{j}:[0, \infty)^{d} \rightarrow[0, \infty)$ are continuously differentiable in each variable, $f_{j}(0, \ldots, 0)=0$, and $f_{j}\left(s_{1}, \ldots, s_{d}\right)>0$ if $s_{i}>0$ for some $i=1, \ldots, d$;

(C2) $f_{j}$ are non-decreasing in each variable;

(C3) $\int_{1}^{\infty}[F(s)]^{-1 / 2} d s=\infty\left(F(s)=\int_{0}^{s} \sum_{i=1}^{d} f_{i}(t, \ldots, t) d t\right)$.

Similar problems to those we are analyzing here are related to steady-state reaction-diffusion, subsonic fluid flows, electric potentials of some bodies and control theory.

\footnotetext{
${ }^{1}$ Supported by CNCSIS-UEFISCDI, project number PN II-IDEI 1080/2008 No. 508/2009.
} 
As an example, the first motivation for studying the above problem stems from the article [8] where the reader observe that such problems arise from the description of the basic stochastic control theory. The controls are to be designed so that the state of the system is constrained to some region. Finding optimal controls is then shown to be equivalent to finding large solutions for a second order semilinear elliptic partial differential equation. In terms of the dynamic programming approach, an explosive solution of (1) corresponds to a value function (or Bellman function) associated to an infinite exit cost (see [8]).

Another motivation comes from the work of [3] where the parabolic problem corresponding to system (1) are models of steady state of non-linear heat conduction through a 2-components mixture.

The numerous applications that lead to favorable answers in order to establish new and significant results for problem (1) as well as the recent results in the field motivate the study of more generally class of problems (1).

The main results of this paper are the following two theorems. They substantially solve the open problem proposed in [7] and complete the results of [2] where only sufficient conditions are obtained.

Theorem 1. Suppose that $(\mathrm{P} 1),(\mathrm{C} 1)-(\mathrm{C} 3)$ are satisfied. If there exists a positive number $\varepsilon$ such that

$$
\int_{0}^{\infty} t^{1+\varepsilon} \sum_{j=1}^{d} \varphi_{j}(t) d t<\infty \quad \text { where } \varphi_{j}(t)=\max _{|x|=t} p_{j}(x),
$$

and $r^{2 N-2} \sum_{j=1}^{d} \varphi_{j}(r)$ is nondecreasing for large $r$, then system (1) has a nonnegative nontrivial bounded solution on $\boldsymbol{R}^{N}$. If, on the other hand, $p_{j}$ satisfy

$$
\int_{0}^{\infty} t \sum_{j=1}^{d} \psi_{j}(t) d t=\infty \quad \text { where } \psi_{j}(t)=\min _{|x|=t} p_{j}(x),
$$

and $r^{2 N-2} \sum_{j=1}^{d} \psi_{j}(r)$ is nondecreasing for large $r$, then system (1) has no nonnegative nontrivial entire bounded radial solution on $\boldsymbol{R}^{N}$.

Theorem 2. Assume that $p_{j}: \boldsymbol{R}^{N} \rightarrow[0, \infty)(j=1, \ldots, d)$ are spherically symmetric continuous functions (i.e. $\left.p_{j}(x)=p_{j}(|x|)\right)$. If $f_{j}(j=1, \ldots, d)$ satisfy $(\mathrm{C} 1)-(\mathrm{C} 3)$, then the problem (1) has a nonnegative nontrivial entire radial solution. Suppose furthermore that $r^{2 N-2} \sum_{j=1}^{d} p_{j}(r)$ is nondecreasing for large $r$. If $p_{j}$ satisfies

$$
\int_{0}^{\infty} \frac{1}{t^{N-1}} \int_{0}^{t} s^{N-1} p_{j}(s) d s d t=\infty \quad \text { for all } j=1, \ldots, d
$$


then any nonnegative nontrivial solution $\left(u_{1}, \ldots, u_{d}\right)$ of $(1)$ is large. Conversely, if (1) has a nonnegative entire large solution, then $p_{j}$ satisfy

$$
\int_{0}^{\infty} r^{1+\varepsilon} \sum_{j=1}^{d} p_{j}(r) d r=\infty
$$

for every $\varepsilon>0$.

\section{Preliminary result}

We recall the following definition of lower and upper solution which are our main tool in the proof of the solvability of problem (1).

Definition 1. A function $\left(w_{1}, \ldots, w_{d}\right) \in\left[C_{l o c}^{2, \alpha}\left(\boldsymbol{R}^{N}\right)\right]^{d}(\alpha \in(0,1))$ is called a lower solution of the problem (1) if

$$
\Delta w_{i} \geq p_{i}(x) f_{i}\left(w_{1}, \ldots, w_{d}\right) \quad \text { in } \boldsymbol{R}^{N} \quad \text { for all } i=1, \ldots, d
$$

Definition 2. We say that $\left(v_{1}, \ldots, v_{d}\right) \in\left[C_{l o c}^{2, \alpha}\left(\boldsymbol{R}^{N}\right)\right]^{d}(\alpha \in(0,1))$ is an upper solution of the problem (1) if

$$
\Delta v_{i} \leq p_{i}(x) f_{i}\left(v_{1}, \ldots, v_{d}\right) \quad \text { in } \boldsymbol{R}^{N} \quad \text { for all } i=1, \ldots, d .
$$

We need the following lemma which can be found in [4, Theorem 5.1, pp. 146]:

Lemma 3. Make the same assumptions on $p_{j}$ and $f_{j}(j=1, \ldots, d)$ as in Theorem 1. If the problem (1) has a pair of upper and lower bounded solutions $\left(v_{1}, \ldots, v_{d}\right)$ and $\left(w_{1}, \ldots, w_{d}\right)$ fulfilling $w_{i}(x) \leq v_{i}(x), i=1, \ldots, d, \forall x \in \boldsymbol{R}^{N}$ then there exists a bounded function $\left(u_{1}, \ldots, u_{d}\right)$ belonging to $\left[C_{l o c}^{2, \alpha}\left(\boldsymbol{R}^{N}\right)\right]^{d}(\alpha \in(0,1))$ with

$$
w_{i}(x) \leq u_{i}(x) \leq v_{i}(x), \quad i=1, \ldots, d, \forall x \in \boldsymbol{R}^{N}
$$

and satisfying (1).

\section{Proofs of main results}

In this section we give the proofs of Theorems 1 and 2.

Proof of the Theorem 1. Assume that (2) holds. In this section, we use the method of upper and lower solutions to show the existence of positive bounded solutions of the problem (1). Our aim is to construct an upper 
solution $\left(v_{1}, \ldots, v_{d}\right)$ and a lower solution $\left(w_{1}, \ldots, w_{d}\right)$ for the problem (1). On this purpose, we first prove the existence of $\left(w_{1}, \ldots, w_{d}\right)$ to the system

$$
\left\{\begin{array}{l}
\Delta w_{1}(r)=\varphi_{1}(r) f_{1}\left(w_{1}, \ldots, w_{d}\right) \quad \text { for } r:=|x|, \\
\cdots \\
\Delta w_{d}(r)=\varphi_{d}(r) f_{d}\left(w_{1}, \ldots, w_{d}\right) \text { for } r:=|x|
\end{array} \text { in } \boldsymbol{R}^{N}\right.
$$

Observe that we can rewrite (6) as follows:

$$
\left\{\begin{array}{l}
\left(r^{N-1} w_{1}(r)\right)^{\prime}=r^{N-1} \varphi_{1}(r) f_{1}\left(w_{1}, \ldots, w_{d}\right) \\
\cdots \\
\left(r^{N-1} w_{d}(r)\right)^{\prime}=r^{N-1} \varphi_{d}(r) f_{d}\left(w_{1}, \ldots, w_{d}\right)
\end{array}\right.
$$

Then radial solutions of (6) are any solution $\left(w_{1}, \ldots, w_{d}\right)$ of the integral equations

$$
\left\{\begin{array}{l}
w_{1}(r)=\frac{1}{d}+\int_{0}^{r} \frac{1}{t^{N-1}} \int_{0}^{t} s^{N-1} \varphi_{1}(s) f_{1}\left(w_{1}(s), \ldots, w_{d}(s)\right) d s d t \\
\ldots \\
w_{d}(r)=\frac{1}{d}+\int_{0}^{r} \frac{1}{t^{N-1}} \int_{0}^{t} s^{N-1} \varphi_{d}(s) f_{d}\left(w_{1}(s), \ldots, w_{d}(s)\right) d s d t
\end{array}\right.
$$

To establish a solution to this system, we use successive approximation. Define sequences $\left\{w_{j}^{k}\right\}_{j=1, \ldots, d}^{k \geq 1}$ on $[0, \infty)$ by

$$
\left\{\begin{array}{l}
w_{1}^{0}=\cdots=w_{d}^{0}=\frac{1}{d}, \quad r \geq 0, \\
w_{i}^{k}(r)=\frac{1}{d}+\int_{0}^{r} \frac{1}{t^{N-1}} \int_{0}^{t} s^{N-1} \varphi_{i}(s) f_{i}\left(w_{1}^{k-1}(s), \ldots, w_{d}^{k-1}(s)\right) d s d t, \quad i=1, \ldots, d .
\end{array}\right.
$$

We remark that, for all $r \geq 0, j=1, \ldots, d$ and $k \in N$

$$
w_{j}^{k}(r) \geq \frac{1}{d}
$$

Moreover, proceeding by induction we conclude $\left\{w_{j}^{k}\right\}_{j=1, \ldots, d}^{k \geq 1}$ are non-decreasing sequence on $[0, \infty)$. We note that $\left\{w_{j}^{k}\right\}_{j=1, \ldots, d}^{k \geq 1}$ satisfy

$$
\left\{\begin{array}{l}
{\left[r^{N-1}\left(w_{1}^{k}\right)^{\prime}\right]^{\prime}=r^{N-1} \varphi_{1}(r) f_{1}\left(w_{1}^{k-1}, \ldots, w_{d}^{k-1}\right)} \\
\cdots \\
{\left[r^{N-1}\left(w_{d}^{k}\right)^{\prime}\right]^{\prime}=r^{N-1} \varphi_{d}(r) f_{d}\left(w_{1}^{k-1}, \ldots, w_{d}^{k-1}\right) .}
\end{array}\right.
$$


By the monotonicity of $\left\{w_{j}^{k}\right\}_{j=1, \ldots, d}^{k \geq 1}$ we have the inequalities

$$
\left\{\begin{aligned}
{\left[r^{N-1}\left(w_{1}^{k}\right)^{\prime}\right]^{\prime} } & =r^{N-1} \varphi_{1}(r) f_{1}\left(w_{1}^{k-1}, \ldots, w_{d}^{k-1}\right) \\
& \leq r^{N-1} \varphi_{1}(r) \sum_{i=1}^{d} f_{i}\left(\sum_{j=1}^{d} w_{j}^{k}(r), \ldots, \sum_{j=1}^{d} w_{j}^{k}(r)\right) \\
\cdots & \\
{\left[r^{N-1}\left(w_{d}^{k}\right)^{\prime}\right]^{\prime} } & =r^{N-1} \varphi_{d}(r) f_{d}\left(w_{1}^{k-1}, \ldots, w_{d}^{k-1}\right) \\
& \leq r^{N-1} \varphi_{d}(r) \sum_{i=1}^{d} f_{i}\left(\sum_{j=1}^{d} w_{j}^{k}(r), \ldots, \sum_{j=1}^{d} w_{j}^{k}(r)\right)
\end{aligned}\right.
$$

Thus, summing up inequalities, we have

$$
\left[r^{N-1} \sum_{i=1}^{d}\left(w_{i}^{k}\right)^{\prime}\right]^{\prime} \leq r^{N-1} \sum_{i=1}^{d} \varphi_{i}(r) \sum_{i=1}^{d} f_{i}\left(\sum_{j=1}^{d} w_{j}^{k}(r), \ldots, \sum_{j=1}^{d} w_{j}^{k}(r)\right) .
$$

Choose $R>0$ so that $r^{2 N-2} \sum_{j=1}^{d} \varphi_{j}(r)$ are non-decreasing for $r \geq R$. We are now ready to show that $w_{j}^{k}(R)$ and $\left(w_{j}^{k}(R)\right)^{\prime}$, both of which are nonnegative, are bounded above independent of $k$. To do this, let

$$
\phi_{j}^{R}=\max \left\{\varphi_{j}(r): 0 \leq r \leq R\right\}, \quad j=1, \ldots, d .
$$

Using this and the fact that $\left(w_{j}^{k}\right)^{\prime} \geq 0$, we note that (7) yields

$$
\left\{\begin{array}{l}
{\left[\left(w_{1}^{k}\right)^{\prime}\right]^{\prime} \leq \phi_{1}^{R} \sum_{i=1}^{d} f_{i}\left(\sum_{i=1}^{d} w_{i}^{k}, \ldots, \sum_{i=1}^{d} w_{i}^{k}\right),} \\
\ldots \\
{\left[\left(w_{d}^{k}\right)^{\prime}\right]^{\prime} \leq \phi_{d}^{R} \sum_{i=1}^{d} f_{i}\left(\sum_{i=1}^{d} w_{i}^{k}, \ldots, \sum_{i=1}^{d} w_{i}^{k}\right) .}
\end{array}\right.
$$

This implies that

$$
\sum_{i=1}^{d}\left[\left(w_{i}^{k}\right)^{\prime}\right]^{\prime} \leq\left(\sum_{i=1}^{d} \phi_{i}^{R}\right) \sum_{i=1}^{d} f_{i}\left(\sum_{i=1}^{d} w_{i}^{k}, \ldots, \sum_{i=1}^{d} w_{i}^{k}\right) .
$$

On the other hand, multiplying this equation by $\left(\sum_{i=1}^{d} w_{i}^{k}\right)^{\prime}$ and integrating we also have

$$
\left[\left(\sum_{i=1}^{d} w_{i}^{k}(r)\right)^{\prime}\right]^{2} \leq 2\left(\sum_{i=1}^{d} \phi_{i}^{R}\right) \int_{1}^{\sum_{i=1}^{d} w_{i}^{k}(r)} \sum_{i=1}^{d} f_{i}(s, \ldots, s) d s, \quad 0 \leq r \leq R
$$

Integrating the above inequality, we see that

$$
\int_{1}^{\sum_{i=1}^{d} w_{i}^{k}(R)}\left[\int_{1}^{t} \sum_{i=1}^{d} f_{i}(s, \ldots, s) d s\right]^{-1 / 2} d t \leq \sqrt{2\left(\sum_{i=1}^{d} \phi_{i}^{R}\right)} R .
$$


It follows from the above relation and by the assumption $\mathrm{C} 3)$ that $\sum_{i=1}^{d} w_{i}^{k}(R)$ is bounded above independent of $k$. Using this fact in (9) shows that the same is true of $\left(\sum_{i=1}^{d} w_{i}^{k}(R)\right)^{\prime}$. Thus, the sequences $w_{i}^{k}(R)$ and $\left(w_{i}^{k}(R)\right)^{\prime}$ are bounded above independent of $k$. Finally, we show that the non-decreasing sequences $w_{i}^{k}$ is bounded for all $r \geq 0$ and all $k$. Multiplying (8) by $r^{N-1}\left(\sum_{i=1}^{d} w_{i}^{k}\right)^{\prime}$ and integrating gives

$$
\begin{aligned}
{\left[r^{N-1}\left(\sum_{i=1}^{d} w_{i}^{k}(r)\right)^{\prime}\right]^{2} } \\
\leq\left[R^{N-1}\left(\sum_{i=1}^{d} w_{i}^{k}(R)\right)^{\prime}\right]^{2} \\
\quad+2 \int_{R}^{r} s^{2 N-2} \sum_{i=1}^{d} \varphi_{i}(s) \sum_{i=1}^{d} f_{i}\left(\sum_{i=1}^{d} w_{i}^{k}, \ldots, \sum_{i=1}^{d} w_{i}^{k}\right)\left(\sum_{i=1}^{d} w_{i}^{k}\right)^{\prime} d s,
\end{aligned}
$$

for $r \geq R$. Noting that, by the monotonicity of $s^{2 N-2} \sum_{i=1}^{d} \varphi_{i}(s)$ for $s \geq R$, we get

$$
\left[r^{N-1}\left(\sum_{i=1}^{d} w_{i}^{k}(r)\right)^{\prime}\right]^{2} \leq C+2 r^{2 N-2} \sum_{i=1}^{d} \varphi_{i}(r) F\left(\sum_{i=1}^{d} w_{i}^{k}(r)\right)
$$

where $C=\left[R^{N-1}\left(\sum_{i=1}^{d} w_{i}^{k}(R)\right)^{\prime}\right]^{2}$, which yields

$$
\left(\sum_{i=1}^{d} w_{i}^{k}\right)^{\prime} \leq \sqrt{C} r^{1-N}+\sqrt{2 \sum_{i=1}^{d} \varphi_{i}(r)}\left[F\left(\sum_{i=1}^{d} w_{i}^{k}(r)\right)\right]^{1 / 2},
$$

and hence

$$
\frac{d}{d r} \int_{\sum_{i=1}^{d} w_{i}^{k}(R)}^{\sum_{i=1}^{d} w_{i}^{k}(r)}[F(t)]^{-1 / 2} d t \leq \sqrt[2]{C} r^{1-N}\left[F\left(\sum_{i=1}^{d} w_{i}^{k}(r)\right)\right]^{-1 / 2}+\left(2 \sum_{i=1}^{d} \varphi_{i}(r)\right)^{1 / 2} .
$$

Integrating this and using the fact that

$$
\begin{aligned}
\left(2 \sum_{i=1}^{d} \varphi_{i}(s)\right)^{1 / 2} & =\left(2 s^{1+\varepsilon} \sum_{i=1}^{d} \varphi_{i}(s) s^{-1-\varepsilon}\right)^{1 / 2} \\
& \leq s^{1+\varepsilon}\left(\sum_{i=1}^{d} \varphi_{i}(s)\right)+s^{-1-\varepsilon}
\end{aligned}
$$


for each $\varepsilon>0$, we have

$$
\begin{aligned}
\int_{\sum_{i=1}^{d} w_{i}^{k}(R)}^{\sum_{i=1}^{d} w_{i}^{k}(r)}[F(t)]^{-1 / 2} d t \leq & \sqrt[2]{C} \int_{R}^{r} t^{1-N}\left[F\left(\sum_{i=1}^{d} w_{i}^{k}(t)\right)\right]^{-1 / 2} d t \\
& +\left[\int_{R}^{r} t^{1+\varepsilon} \sum_{i=1}^{d} \varphi_{i}(t) d t+\int_{R}^{r} t^{-1-\varepsilon} d t\right] \\
\leq & \sqrt[2]{C}\left[F\left(\sum_{i=1}^{d} w_{i}^{k}(R)\right)\right]^{-1 / 2} \int_{R}^{r} t^{1-N} d t \\
& +\left[\int_{R}^{r} t^{1+\varepsilon}\left(\sum_{i=1}^{d} \varphi_{i}(t)\right) d t+\frac{1}{\varepsilon R^{\varepsilon}}\right]
\end{aligned}
$$

The above relation is needed in proving the bounded of the function $\left\{w_{j}^{k}\right\}_{j=1, \ldots, d}^{k \geq 1}$ in the following. Indeed, since for each $\varepsilon>0$ the right side of this inequality is bounded independent of $k$ (note that $w_{i}^{k}(t) \geq 1 / d$ for all $i=1, \ldots, d$ ), so is the left side and hence, in light of C3), the sequence $\left\{\sum_{i=1}^{d} w_{i}^{k}\right\}^{k \geq 1}$ is a bounded sequence and so $\left\{w_{j}^{k}\right\}_{j=1, \ldots, d}^{k \geq 1}$ are bounded sequence. Thus $\left\{w_{j}^{k}\right\}_{j=1, \ldots, d}^{k \geq 1}$ $\rightarrow\left\{w_{j}\right\}_{j=1, \ldots, d}$ as $k \rightarrow \infty$ and the limit functions $\left\{w_{j}\right\}_{j=1, \ldots, d}$ are positive entire solutions of system (6). It is obvious that every solution of (6) is a lower solution to (1).

We let $M$ be the least upper bound of $\sum_{i=1}^{d} w_{i}$ and note that

$$
M=\lim _{r \rightarrow \infty} \sum_{i=1}^{d} w_{i}(r) .
$$

Now let $\psi_{i}(t)=\min _{|x|=t} p_{i}(x) \quad(i=1, \ldots, d)$ and $\left\{v_{j}\right\}_{j=1, \ldots, d}$ be the positive increasing bounded solutions of

$$
\left\{\begin{array}{l}
v_{1}(r)=M+\int_{0}^{r} \frac{1}{t^{N-1}} \int_{0}^{t} s^{N-1} \psi_{1}(s) f_{1}\left(v_{1}(s), \ldots, v_{d}(s)\right) d s d t \\
\cdots \\
v_{d}(r)=M+\int_{0}^{r} \frac{1}{t^{N-1}} \int_{0}^{t} s^{N-1} \psi_{d}(s) f_{d}\left(v_{1}(s), \ldots, v_{d}(s)\right) d s d t
\end{array}\right.
$$

which, of course, satisfies $(6)$ with $w_{j}(j=1, \ldots, d)$ replaced with $v_{j}(j=1, \ldots, d)$ and $\varphi_{j}(j=1, \ldots, d)$ replaced with $\psi_{j}(j=1, \ldots, d)$. It is also clear that $v_{j} \geq M$. If we modify the proof of the lower solution $\left(w_{1}, \ldots, w_{d}\right)$ we obtain the existence of an upper solution $\left(v_{1}, \ldots, v_{d}\right)$. Hence the standard upper-lower solution principle implies that $(1)$ has a solution $\left(u_{1}, \ldots, u_{d}\right)$ such that

$$
w_{i}(x) \leq u_{i}(x) \leq v_{i}(x), \quad i=1, \ldots, d,
$$

(see Lemma 3). 
Now assume that (3) holds. Assume to the contrary that there exist a nonnegative nontrivial entire bounded radial solution $\left(u_{1}, \ldots, u_{d}\right)$ on $\boldsymbol{R}^{N}$ for the system (1). Assuming $M_{i}=\sup _{x \in \boldsymbol{R}^{N}} u_{i}(x)(i=1, \ldots, d)$ and knowing that $u_{i}^{\prime} \geq 0$, we get $\lim _{r \rightarrow \infty} u_{i}=M_{i}$. Thus there exists $R>0$ such that $u_{i} \geq M_{i} / 2$ $(i=1, \ldots, d)$ for $r \geq R$. From conditions of $f_{i}$, it follows that

$$
f_{i}\left(u_{1}, \ldots, u_{d}\right) \geq f_{i}\left(M_{1} / 2, \ldots, M_{d} / 2\right):=c_{0}^{i}, \quad \text { for } r \geq R \text { and } i=1, \ldots, d .
$$

Using this we get the following

$$
\begin{gathered}
u_{1} \geq u_{1}(0)+\int_{0}^{r} t^{1-N} \int_{0}^{t} s^{N-1} \psi_{1}(s) f_{1}\left(u_{1}(s), \ldots, u_{d}(s)\right) d s d t \\
\ldots \\
u_{d} \geq u_{d}(0)+\int_{0}^{r} t^{1-N} \int_{0}^{t} s^{N-1} \psi_{d}(s) f_{d}\left(u_{1}(s), \ldots, u_{d}(s)\right) d s d t .
\end{gathered}
$$

Rearranging the terms, and by using these conditions in (12) follows

$$
\sum_{i=1}^{d} u_{i}(r) \geq d m_{1}+m_{2} \frac{1}{N} \int_{R}^{r} t \sum_{i=1}^{d} \psi_{i}(t) d t \rightarrow \infty \quad \text { as } r \rightarrow \infty,
$$

where $m_{1}=\min \left\{u_{1}(0), \ldots, u_{d}(0)\right\}$ and $m_{2}:=\min \left\{c_{0}^{1}, \ldots, c_{0}^{d}\right\}$. A contradiction to the boundedness of $\sum_{i=1}^{d} u_{i}(r)$. This proves Theorem 1 .

Proof of the Theorem 2. It is known from [6, 12, Theorem 2] that the problem

$$
\Delta z(r)=\sum_{i=1}^{d} p_{i}(r) \sum_{i=1}^{d} f_{i}(z(r), \ldots, z(r)) \quad \text { for } r:=|x|, \quad x \in \boldsymbol{R}^{N}
$$

has a non-negative non-trivial entire solution. Moreover, for each $R>0$, there exists $c_{R}>0$ such that $z(R) \leq c_{R}$. Due to the fact that $z$ is radial, we have

$$
z(r)=z(0)+\int_{0}^{r} \frac{1}{t^{N-1}} \int_{0}^{t} s^{N-1} \sum_{i=1}^{d} p_{i}(s) \sum_{i=1}^{d} f_{i}(z(s), \ldots, z(s)) d s d t \quad \text { for all } r \geq 0 .
$$

We choose $\beta_{1} \in(0, z(0)]$. Define the sequences $\left\{u_{j}^{k}\right\}_{j=1, \ldots, d}^{k \geq 1}$ on $[0, \infty)$ by

$$
\left\{\begin{array}{l}
u_{1}^{0}=\cdots=u_{d}^{0}=\beta_{1} \quad \text { for all } r \geq 0 \\
u_{1}^{k}(r)=\beta_{1}+\int_{0}^{r} \frac{1}{t^{N-1}} \int_{0}^{t} s^{N-1} p_{1}(s) f_{1}\left(u_{1}^{k-1}(s), \ldots, u_{d}^{k-1}(s)\right) d s d t, \\
\cdots \\
u_{d}^{k}(r)=\beta_{1}+\int_{0}^{r} \frac{1}{t^{N-1}} \int_{0}^{t} s^{N-1} p_{d}(s) f_{d}\left(u_{1}^{k-1}(s), \ldots, u_{d}^{k-1}(s)\right) d s d t, \quad d \geq 1 .
\end{array}\right.
$$


With the same arguments as in the proof of Theorem 1 we obtain that $\left\{u_{j}^{k}\right\}_{j=1, \ldots, d}^{k \geq 1}$ are non-decreasing sequence on $[0, \infty)$. Because $z^{\prime}(r) \geq 0$ follows $0<\beta_{1} \leq z(0) \leq z(r)$ for all $r \geq 0$ and so

$$
\begin{aligned}
u_{1}^{1}(r) & =\beta_{1}+\int_{0}^{r} \frac{1}{t^{N-1}} \int_{0}^{t} s^{N-1} p_{1}(s) f_{i}\left(u_{1}^{0}(s), \ldots, u_{d}^{0}(s)\right) d s d t \\
& \leq z(0)+\int_{0}^{r} \frac{1}{t^{N-1}} \int_{0}^{t} s^{N-1} \sum_{i=1}^{d} p_{i}(s) \sum_{i=1}^{d} f_{i}(z(s), \ldots, z(s)) d s d t=z(r) .
\end{aligned}
$$

Thus $u_{1}^{1}(r) \leq z(r)$. Similar arguments show that

$$
u_{j}^{k} \leq z(r)(j=1, \ldots, d) \quad \text { for all } r \in[0, \infty) \text { and } k \geq 1 .
$$

Thus, for every $x \in \boldsymbol{R}^{N}$ the sequence $u_{j}^{k}(|x|)(j=1, \ldots, d)$ have subsequences, denoted again by $u_{j}^{k}(|x|)(j=1, \ldots, d)$, converging and

$$
\left(u_{1}(|x|), \ldots, u_{d}(|x|)\right):=\left(\lim _{k \rightarrow \infty} u_{1}^{k}(|x|), \ldots, \lim _{k \rightarrow \infty} u_{d}^{k}(|x|)\right),
$$

is an entire radial solution of system (1).

Let $\left(u_{1}, \ldots, u_{d}\right)$ be any non-negative non-trivial entire radial solution of $(1)$ and suppose that $p_{j}(j=1, \ldots, d)$ satisfies (4). Since $u_{i}(i=1, \ldots, d)$ is nontrivial and non-negative, there exists $R>0$ so that $u_{i}(R)>0$. Since $u_{i}^{\prime} \geq 0$, we get $u_{i}(r) \geq u_{i}(R)$ for $r \geq R$ and thus from

$$
\left\{\begin{array}{l}
u_{1}(r)=u_{1}(0)+\int_{0}^{r} \frac{1}{t^{N-1}} \int_{0}^{t} s^{N-1} p_{1}(s) f_{1}\left(u_{1}(s), \ldots, u_{d}(s)\right) d s d t \\
\ldots \\
u_{d}(r)=u_{d}(0)+\int_{0}^{r} \frac{1}{t^{N-1}} \int_{0}^{t} s^{N-1} p_{d}(s) f_{d}\left(u_{1}(s), \ldots, u_{d}(s)\right) d s d t
\end{array}\right.
$$

we get

$$
\left\{\begin{aligned}
u_{i}(r) & =u_{i}(0)+\int_{0}^{r} \frac{1}{t^{N-1}} \int_{0}^{t} s^{N-1} p_{i}(s) f_{i}\left(u_{1}(s), \ldots, u_{d}(s)\right) d s d t, \quad i=1, \ldots, d \\
& \geq u_{i}(R)+f_{i}\left(u_{1}(R), \ldots, u_{d}(R)\right) \int_{R}^{r} \frac{1}{t^{N-1}} \int_{R}^{t} s^{N-1} p_{i}(s) d s d t \rightarrow \infty \quad \text { as } r \rightarrow \infty .
\end{aligned}\right.
$$

Conversely, if $f_{i}(i=1, \ldots, d)$ satisfy $(\mathrm{C} 1)-(\mathrm{C} 3)$ and $\left(w_{1}, \ldots, w_{d}\right)$ is a nonnegative entire large solution of (1), then $w_{i}$ satisfy

$$
\left\{\begin{array}{l}
{\left[r^{N-1}\left(w_{1}\right)^{\prime}\right]^{\prime}=p_{1}(x) f_{1}\left(w_{1}, \ldots, w_{d}\right)} \\
\cdots \\
{\left[r^{N-1}\left(w_{d}\right)^{\prime}\right]^{\prime}=p_{d}(x) f_{d}\left(w_{1}, \ldots, w_{d}\right)}
\end{array}\right.
$$


Then, using the monotonicity of $r^{2 N-2} \sum_{j=1}^{d} p_{j}(r)$ we can apply similar arguments used in obtaining Theorem 1 to get

$$
\left(\sum_{i=1}^{d} w_{i}^{k}(r)\right)^{\prime} \leq \sqrt{C} r^{1-N}+\sqrt{2 \sum_{i=1}^{d} \varphi_{i}(r)} \sqrt{F\left(\sum_{i=1}^{d} w_{i}^{k}(r)\right)} .
$$

This conclusion and relation (11) imply that

$$
\begin{aligned}
\int_{\sum_{i=1}^{d} w_{i}(R)}^{\sum_{i=1}^{d} w_{i}(r)}[F(t)]^{-1 / 2} d t \leq & \frac{\sqrt[2]{C}}{\left[F\left(\sum_{i=1}^{d} w_{i}(R)\right)\right]^{1 / 2}} \int_{R}^{r} t^{1-N} d t \\
& +\left(\int_{R}^{r} t^{1+\varepsilon} \sum_{i=1}^{d} p_{i}(t) d t+\frac{1}{\varepsilon R^{\varepsilon}}\right) \\
\leq & C_{R}+\int_{R}^{r} t^{1+\varepsilon} p_{i}(t) d t
\end{aligned}
$$

where

$$
C_{R}=\sqrt[2]{C}\left[F\left(\sum_{i=1}^{d} w_{i}(R)\right)\right]^{-1 / 2} \frac{R^{N-2}}{N-2}+\frac{1}{\varepsilon R^{\varepsilon}} .
$$

By taking $r \rightarrow \infty$ in (14) we obtain that $p_{j}(j=1, \ldots, d)$ satisfies (5). This completes the proof of the theorem.

We conclude this paper with some remarks:

Remark 1. The time-independent Schrödinger equation in quantum mechanics is

$$
\left(h^{2} / 2 m\right) \Delta u=(V-E) u
$$

where $h=6.625 \cdot 10^{-27} \mathrm{erg}$ sec is the Planck constant, $m$ is the mass of a particle moving under the action of a force field described by the potential $V(x, y, z)$ whose wave function is $u(x, y, z, t)$ and the quantity $E$ is the total energy of the particle, problems which falls into the class of equations discussed here.

Remark 2. If $(\mathrm{C} 1)-(\mathrm{C} 3)$ are satisfied then

$$
\int_{1}^{\infty}\left(\int_{0}^{t} f_{i}(s, \ldots, s)\right)^{-1 / 2} d s d t=\infty \quad \text { for all } i=1, \ldots, d .
$$

Remark 3 (see [1]). If C1)-C2) and

$$
\int_{1}^{\infty}\left(\sum_{i=1}^{d} f_{i}(s, \ldots, s)\right)^{-1} d s=\infty
$$


are satisfied, then

$$
\int_{1}^{\infty}\left(\int_{0}^{t} \sum_{i=1}^{d} f_{i}(s, \ldots, s)\right)^{-1 / 2} d s d t=\infty
$$

Acknowledgement. The author would like to thank to the editors and reviewers for valuable comments and suggestions which contributed to improve this article.

\title{
References
}

[ 1 ] Covei, D. P., Large and Entire Large Solution for a Quasilinear Problem, Nonlinear Anal., 70 (2009), 1738-1745.

[2] Covei, D. P., Existence of entire radially symmetric solutions for a quasilinear system with $d$-equations, Hacet. J. Math. Stat., 40 (2011), 433-439.

[ 3 ] Escobedo, M. and Herrero, M. M., Boundedness and blow up for a semilinear reactiondiffusion system, J. Differential Equations, 89 (1991), 176-202.

[4] Kawano, N., On bounded entire solutions of semilinear elliptic equations, Hiroshima Math. J., 14 (1984), 125-158.

[5] Keller, J. B., On solutions of $\Delta u=f(u)$, Comm. Pure Appl. Math., 10 (1957), 503-510.

[6] Lair, A. V., Large solutions of semilinear elliptic equations under the Keller-Osserman condition, J. Math. Anal. Appl., 328 (2007), 1247-1254.

[7] Lair, A. V., A necessary and sufficient condition for the existence of large solutions to sublinear elliptic systems, J. Math. Anal. Appl., 365 (2010), 103-108.

[8] Lasry, J. M. and Lions, P. L., Nonlinear Elliptic Equations with Singular Boundary conditions and Stochastic Control with State Constraints, Math. Ann., 283 (1989), 583-630.

[9] Miao, Q. and Yang, Z., On the existence of multiple positive entire solutions for a quasilinear elliptic systems, Appl. Math. Comput., 198 (2008), 12-23.

[10] Pohožaev, S. I., The Dirichlet problem for the equation $\Delta u=u^{2}$, Dokl. Akad. Nauk. USSR, 134 (1960), 769-772. English translation: Soviet Math. Dokl., 1 (1960), 1143-1146.

[11] Osserman, R., On the inequality $\Delta u \geq f(u)$, Pacific J. Math., 7 (1957), 1641-1647.

[12] Yuan, J. and Yang, Z., Existence of large solutions for a class of quasilinear elliptic equations, Appl. Math. Comput., 201 (2008), 852-858.

\author{
nuna adreso: \\ Constantin Brâncuşi University of Târgu-Jiu \\ Str. Grivitei, Nr. 1, Târgu-Jiu, Gorj \\ România \\ E-mail: coveid@yahoo.com
}

(Ricevita la 13-an de junio, 2010)

(Reviziita la 17-an de oktobro, 2010) 